be influenced by relatively small sample size and the early detection of haemochromatosis with the aid of genetic analysis, allowing initiation of venesection before development of advanced disease. Further studies using prospective recruitment of patients for LSM at the point of diagnosis alongside biopsy to stage fibrosis would allow a more complete evaluation of LSM performance.

\section{P071 SILYMARIN-CHOLINE COMBINATION VERSUS URSODEOXYCHOLIC ACID IN NON-ALCOHOLIC FATTY LIVER DISEASE: A RANDOMISED DOUBLE-BLIND CLINICAL TRIAL}

\begin{abstract}
${ }^{1}$ Purba Chakrabarty*, ${ }^{2}$ Saubhik Ghosh, ${ }^{3}$ Mayukh Mukherjee, ${ }^{4}$ Rajasee Adhikary, ${ }^{5}$ Shatavisa Mukherjee, ${ }^{4}$ Souvik Majumder, ${ }^{4}$ Dipak Kumar Sarkar, ${ }^{6}$ Arunansu Talukdar. ${ }^{1}$ Walsh, SDH, Serampore, India, Serampore, Hooghly, India; ${ }^{2}$ Department of Medical Gastroenterology, Medical College and Hospital, Kolkata, Kolkata, India; ${ }^{3}$ Department of Pharmacology, R. G. Kar Medical College and Hospital, Kolkata, Kolkata, India; ${ }^{4}$ Department of Pharmacology, Medical College and Hospital, Kolkata, Kolkata, India; ${ }^{5}$ ADR Monitoring Centre, School of Tropical Medicine, Kolkata, Kolkata, India; ${ }^{6}$ Department of Medicine, Medical College and Hospital, Kolkata, Kolkata, India
\end{abstract}

\subsection{6/gutjnl-2021-BASL.80}

Introduction Prevalence of Non-Alcoholic Fatty Liver Disease (NAFLD) is $20 \%-30 \%$ and $5 \%-18 \%$ in Western countries and Asia respectively. ${ }^{1}$ Currently there is no evidence-based standard of care. Since an oxidative stress and dietary deficiency of choline have been implicated in the pathophysiology of hepatic insult, the use of natural compounds like Silymarin, Choline and Ursodeoxycholic acid (UDCA) represents an extremely popular therapeutic option for the treatment of NAFLD. ${ }^{2}$

Objective To compare the efficacy, safety profile and adherence of Silymarin-Choline combination vis - a - vis UDCA in patients with NAFLD

Method The study was a double blind parallel arm trial where 88 NAFLD diagnosed patients, abiding by the inclusion and exclusion criteria, were randomised to receive either Tablet Silymarin (140 mg) - Choline bitartrate (450 mg) 1 tablet thrice daily or UDCA $(300 \mathrm{mg}) 1$ tablet twice daily for 6 months. Lifestyle modification was advised. Participants were monitored for weight, liver function test, lipid profile parameters, HOMA-IR, liver stiffness measurement and liver biopsy at baseline and 6 months of medication. Monitoring of associated adverse events and adherence were done. Results were tabulated and statistically analyzed for any significant inter or intra group differences using standard statistical software.

Results A total of 39 patients received tablet Silymarin Choline bitartrate (group A) as compared to 40 who received UDCA (group B). Both the groups were comparable at baseline with regard to age (mean \pm SD) [39.33 \pm 9.39 vs 40.63 \pm 10.63 , weight $($ mean \pm SD) $[72.80 \pm 4.24$ vs $72.95 \pm 4.22]$ $\mathrm{Kg}$, BMI $($ mean \pm SD) $[29.11 \pm 1.02$ vs $29.32 \pm 2.07] \mathrm{Kg} / \mathrm{m} 2$, LFT (Alanine aminotransferase, median \pm IQR) $[86.00 \pm 18.00$ vs $87.00 \pm 19.00] \mathrm{IU} / \mathrm{L}$, Lipid Parameters, HOMA-IR (mean $\pm \mathrm{SD})[1.42 \pm 0.53$ vs $1.27 \pm 0.71]$, transient elastography $($ mean $\pm S D) \quad[7.67 \pm 0.97$ vs $7.61 \pm 0.66]$ and tolerability. At 2nd visit, significant individual intra-group reduction in weight, liver and lipid parameters, improvement in transient elastography, liver biopsy, HOMA IR score was noted. Notably, independent $\mathrm{T}$ test suggests significant decrease $(\mathrm{p}<0.05)$ in weight [67.23 \pm 5.87 vs $70.70 \pm 5.11$, p. 0.006], transient elastography [6.32 \pm 1.26 vs $6.85 \pm 0.98$, p. 0.043], HOMA IR score $[0.59 \pm 0.28$ vs $0.77 \pm 0.21$, p. 0.002] more in group A compared to group B.

Conclusion NAFLD is an important cause of liver disease burden across the world. The accepted treatment protocol is to treat the associated comorbidities which cannot stop the progression of disease at times. Tablet Silymarin - Choline bitartrate is effective and tolerable as UDCA when combined with lifestyle modification. Tablet Silymarin - Choline bitartrate can be utilized as an alternative to UDCA in the treatment of NAFLD.

\section{REFERENCES}

1. Hellerbrand C, Schattenberg JM, Peterburs P, Lechner A, Brignoli R. The potential of silymarin for the treatment of hepatic disorders. Clin Phytoscience 2017 Jan $1 ; 2(1): 7$

2. Hajiaghamohammadi AA, Ziaee A, Oveisi S, Masroor H. Effects of metformin, pioglitazone, and silymarin treatment on non-alcoholic fatty liver disease: a randomized controlled pilot study. Hepat Mon [Internet] 2012 Aug 3 [cited 2018 Jun 23];12(8). Available from: https://www.ncbi.nlm.nih.gov/pmc/articles/ PMC3475019/

\section{P072 SYSTEMATIC REVIEW AND META-ANALYSIS OF THE PREVALENCE OF ALCOHOL RELATED LIVER DISEASE}

${ }^{1}$ Sachin Amonker*, ${ }^{1}$ Aryo Houshmand, 'Ian Rowe, ${ }^{2}$ Richard Parker. 'University of Leeds, Leeds, UK; ${ }^{2}$ Leeds Liver Unit, Leeds, UK

\subsection{6/gutjnl-2021-BASL.81}

Introduction Alcohol related liver disease (ALD) is a major cause of morbidity and mortality. The aim of this systematic review and meta-analysis was to define the prevalence of ALD.

Methods A systematic review was undertaken using the search terms ((alcoholic liver disease) AND prevalence OR epidemiology)) in PubMed and Embase databases. Reference lists of the included papers and citing literature were also searched for relevant papers. Single-proportion random-effect meta-analysis was done using the 'meta' package in R. Planned sub-group analyses were done in populations of persons with alcohol use disorder (AUD), for different methods of diagnosis and by geographical region. For the purpose of this review we considered the overall prevalence of all forms of ALD, and the prevalence of alcohol-associated fatty liver (AFL) and alcoholassociated cirrhosis (AC).

Results The literature search identified 7040 studies. After review, 37 papers were included. ALD was defined by biochemistry in 9 studies, by biopsy in 5 studies and by ultrasound in 13 studies. Ten studies used coding from clinical records to define disease.

The overall prevalence of any form of ALD in unselected populations was $4.5 \%$ (95\% confidence interval $3.1-6.3 \%$ ). The prevalence of AFL was 6.3\% (3.2 - 12.1), and prevalence of AC was $0.2 \%(0.1-0.3 \%)$. In populations with AUD, the prevalence of ALD, AFL and AC were 65\% (53.3 - 75.8\%), $17.4 \%(15.3-19.7 \%)$ and $14 \%(5.9-29.9 \%)$ respectively. Studies using ultrasound reported higher prevalence of disease. European cohorts tended to have higher prevalence of disease. Conclusion This systematic review estimates prevalence of ALD at approximately 1 in 20 people, and prevalence of AC as 1 person in 500. Prevalence varies by geography and by diagnostic modality. The higher prevalence of ALD amongst cohorts with AUD suggests that methods for early detection of liver disease are likely to be better deployed in high risk groups. 